\title{
L1506: a prestellar core in the making ${ }^{\star}$
}

\author{
L. Pagani ${ }^{1}$, I. Ristorcelli ${ }^{2}$, N. Boudet ${ }^{2}$, M. Giard ${ }^{2}$, A. Abergel ${ }^{3}$, and J.-P. Bernard ${ }^{2}$ \\ 1 LERMA \& UMR 8112 du CNRS, Observatoire de Paris, 61 Av. de l'Observatoire, 75014 Paris, France \\ e-mail: laurent.pagani@obspm.fr \\ 2 CESR \& UMR 5187 du CNRS/Université de Toulouse, 9 Av. du Colonel Roche, BP 4346, 31028 Toulouse Cedex 4, France \\ e-mail: [ristorcelli; giard; bernard]@cesr.fr \\ 3 IAS, Bât. 121, Université Paris-Sud, 91435 Orsay, France \\ e-mail: alain. abergel@ias.u-psud.fr
}

Received 9 July 2009 / Accepted 2 December 2009

\section{ABSTRACT}

\begin{abstract}
Context. Exploring the structure and dynamics of cold starless clouds is necessary to understand the different steps leading to the formation of protostars. Because clouds evolve slowly, many of them must be studied in detail to identify different moments in a cloud's lifetime.

Aims. We study a fragment of the long filament L1506 in the Taurus region, which we name L1506C, a core with interesting dust properties observed by the PRONAOS balloon-borne telescope.

Methods. To trace the mass content of L1506C and its kinematics, we mapped the dust emission, and the line emission of two key species, $\mathrm{C}^{18} \mathrm{O}$ and $\mathrm{N}_{2} \mathrm{H}^{+}$. We also observed ${ }^{13} \mathrm{CO}$ and $\mathrm{C}^{17} \mathrm{O}$. We model the species emission using 1D Monte Carlo models.

Results. This cloud is reminiscent of $\mathrm{L} 1498$ but also exhibits peculiar features: i) a large envelope emitting solely in ${ }^{13} \mathrm{CO}$ that contains a much smaller core with strong $\mathrm{C}^{18} \mathrm{O}$ depletion at its centre despite a low maximum opacity $\left(A_{\mathrm{V}} \sim 20\right.$ mag); ii) extremely narrow $\mathrm{C}^{18} \mathrm{O}$ lines indicating a low, non-measurable turbulence; iii) contraction traced by $\mathrm{C}^{18} \mathrm{O}$ itself (plus rotation); iv) unexpectedly, the kinematical signature of the external envelope is opposite to that of the core, the ${ }^{13} \mathrm{CO}$ and $\mathrm{C}^{18} \mathrm{O}$ velocity gradients having opposite directions and the $\mathrm{C}^{18} \mathrm{O}$ line profile being blue peaked in contrast to the ${ }^{13} \mathrm{CO}$ one which is red peaked. The core is large $(r=$ $\left.3 \times 10^{4} \mathrm{AU}\right)$ and not very dense $\left(n\left(\mathrm{H}_{2}\right) \leq 5 \times 10^{4} \mathrm{~cm}^{-3}\right.$, possibly less $)$. This core is therefore not yet prestellar.

Conclusions. All these properties imply that the core is kinematically detached from its envelope and in the process of forming a prestellar core. This is the first time that the dynamical formation of a prestellar core has been witnessed. The extremely low turbulence could explain the strong depletion of this core despite its relatively low density and opacity in contrast to undepleted cores such as L1521E, which exhibits a turbulence at least 4 times as high.
\end{abstract}

Key words. stars: formation - ISM: clouds - ISM: abundances - ISM: molecules - ISM: structure - ISM: individual objects: L1506

\section{Introduction}

On their way to forming stars, clouds go through a contraction phase leading to the formation of prestellar cores $(n \geq$ $1 \times 10^{5} \mathrm{~cm}^{-3}$, Keto \& Caselli 2008), which are usually identified by their lack of internal heating sources, their large $\mathrm{CO}$ depletion, and their strong $\mathrm{NH}_{3}$ and $\mathrm{N}_{2} \mathrm{H}^{+}$emission lines for which turbulence is often subthermal. These cores subsequently collapse to form protostars. Many details pertaining to these two condensation steps remain obscure and we do not understand the conditions necessary to produce stars of different masses, high or low, the conditions necessary to initiate the contraction of the cloud or the collapse of the prestellar core, nor the rôle of the magnetic field in supporting the clouds against collapse. Obviously, since the evolution time is long, one has to observe many clouds as a substitute to tracking any single cloud in the process of forming a star. For each cloud, physical and chemical properties should be studied in detail to assess all important parameters such as temperature, density, kinematics, and their respective gradients, if any, and also chemical abundances by volume. By accumulating this type of studies, we can hope to evaluate the impact of cloud age or density upon the depletion

* Based on observations made with the IRAM-30 m. IRAM is supported by INSU/CNRS (France), MPG (Germany), and IGN (Spain). phenomenon, distinguish contraction from oscillation, and describe how prestellar cores and protostars form.

Many prestellar cores have been identified and some have already been studied in detail, including B68, L183, L1498, and L1544. Among these objects, a few cases have been identified as early prestellar cores if their density is already higher than $1 \times 10^{5} \mathrm{~cm}^{-3}$, or simple cores probably about to form prestellar cores if not. The first type includes L1495B, L1521B, and L1521E (Hirota et al. 2002, 2004; Tafalla \& Santiago 2004), which, despite having a high density, show no sign of depletion (strong $\mathrm{CO}$ lines, weak or absent $\mathrm{NH}_{3}$ and $\mathrm{N}_{2} \mathrm{H}^{+}$lines) and are considered to be both physically and chemically young, while the second type is represented by two cases presently, L1498 (Tafalla et al. 2004; Shirley et al. 2005) and the very dense globule G2 in the Coalsack complex (Lada et al. 2004). In the study by Shirley et al. (2005), L1498 exhibits a low density $\left(1-3 \times 10^{4} \mathrm{~cm}^{-3}\right)$ and molecular depletion (as already reported by several other authors, especially Willacy et al. 1998, who, by studying $\mathrm{L} 1498$, reported the first $\mathrm{C}^{18} \mathrm{O}$ depletion in a prestellar core by comparison with a dust map). The $\mathrm{G} 2$ gas content has not yet been studied in detail and only its peculiar, ring-like dust shape indicates its unstable nature despite its low density. This core is expected to turn into a prestellar core (Lada et al. 2004). In this second category, which is considered to represent cores 
that are physically young but chemically evolved, we present a newcomer, a fraction of the L1506 filament.

The cloud that we present in this paper, L1506, attracted attention during a large-scale ${ }^{13} \mathrm{CO}$ survey (Abergel et al. 1994) because of its low (though not unique) $\operatorname{DIRBE}^{1} I_{140 \mu \mathrm{m}} / I_{240 \mu \mathrm{m}}$ and IRAS ${ }^{2} I_{60 \mu \mathrm{m}} / I_{100 \mu \mathrm{m}}$ colour ratios to which the ${ }^{13} \mathrm{CO}$ maps were compared. Its dust properties were studied later on using the PRONAOS ${ }^{3}$ balloon-borne experiment continuum emission data in the submillimetre range $(200-600 \mu \mathrm{m})$ (Stepnik et al. 2003), albeit with a limited angular resolution (2-3.5'). A significant change in the dust properties was detected between the diffuse and the dense part of the cloud: no emission from transiently heated small particles and a strong enhancement (by a factor of 3.4) in the submillimetre emissivity of the latter. This was interpreted as the signature of dust coagulation leading to the formation of fluffy aggregates (consisting of a mixture of very small and large grains). To understand this evolution and investigate the physical conditions associated with efficient aggregate formation (ice mantles, level of turbulence, densities, ...), we concentrate on the gas properties. It is presently clear that depletion plays a key rôle in the gas phase of dark clouds and basically two species are needed to map the gas inside the clouds, namely $\mathrm{C}^{18} \mathrm{O}$ and $\mathrm{N}_{2} \mathrm{H}^{+}$. We may also use $\mathrm{NH}_{3}$ as a tracer but $\mathrm{N}_{2} \mathrm{H}^{+}$ has some advantages over $\mathrm{NH}_{3}$ among which the possibility to use the same telescope as for $\mathrm{C}^{18} \mathrm{O}$, giving a similar resolution and beam correction and also superior diagnostic capabilities as discussed by Pagani et al. (2007). By observing these species, both the undepleted (outer) and the depleted (inner) cores can be traced and their kinematics and physical properties can be modeled. With ${ }^{13} \mathrm{CO}$, we are also able to trace a fraction of the extended envelope. We present and model these observations in this paper and discuss the properties of this cloud.

\section{Observations}

All observations were performed using the IRAM-30 m telescope $^{4}$. The dark nebula L1506 is an elongated object (Onishi et al. 1996; Nercessian et al. 1988) and the SIMBAD ${ }^{5}$ reference position $\left(\alpha_{2000}=4^{\mathrm{h}} 18^{\mathrm{m}} 31.1^{\mathrm{s}} \delta_{2000}=+25^{\circ} 19^{\prime} 25^{\prime \prime}\right)$ is outside the part of the cloud that we present here, which is centred on the PRONAOS emission peak $\left(\alpha_{2000}=4^{\mathrm{h}} 18^{\mathrm{m}} 50^{\mathrm{s}} \delta_{2000}=\right.$ $+25^{\circ} 19^{\prime} 15^{\prime \prime}$, Stepnik et al. 2003) that we propose to name L1506C (A and B are already defined, Lee \& Myers 1999). Its distance is estimated to be 140 pc (Elias 1978; Kenyon et al. 1994).

In September 2004, we mapped the filament dust emission at $1.2 \mathrm{~mm}$ with the MAMBO ${ }^{6}$ II bolometre. Skydips, pointings, and calibration source observations were regularly performed, and data reduction was executed using IRAM proprietary software. The cold filament studied by Stepnik et al. (2003) is clearly visible at the centre (Fig. 1). Spectroscopic observations were performed over several runs: 29 and 30 November 2003, 22 and 23 May 2004, 28 July 2004, and 18 July 2008. In all

\footnotetext{
${ }_{1}$ Diffuse Infra Red Background Experiment, http: //lambda.gsfc. nasa.gov

2 Infra Red Astronomical Satellite, http://lambda.gsfc.nasa. gov

3 PROgramme NAtional d'Observations Submillimétriques.

${ }^{4}$ http://www.iram-institute.org/

5 http://simbad.u-strasbg.fr/sim-fid.pl

${ }^{6}$ MAx-Planck-Millimeter-BOlometer, see IRAM website.
}

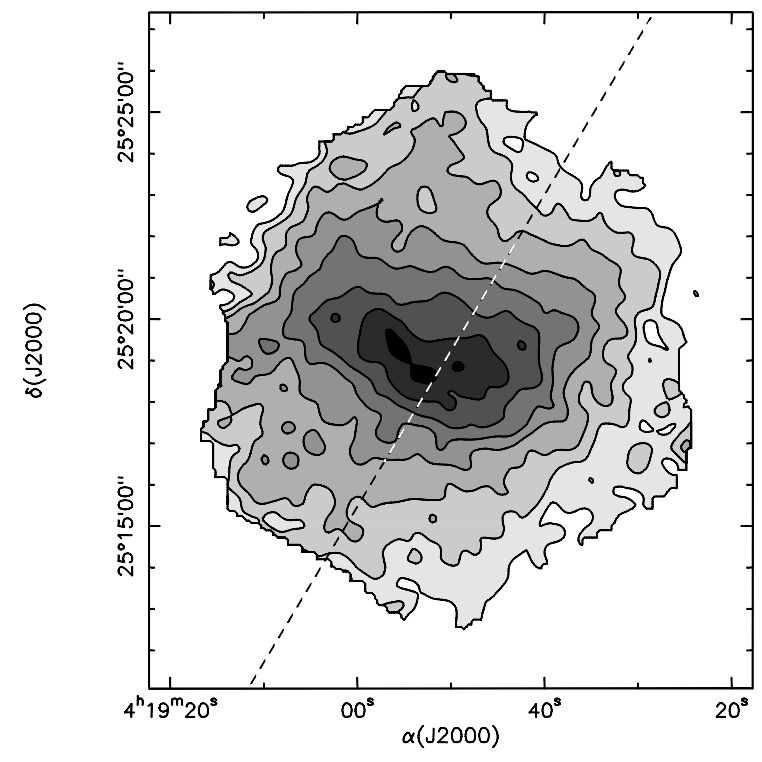

Fig. 1. Dust emission at $1.2 \mathrm{~mm}$ as measured by MAMBO II. Resolution was degraded to $30^{\prime \prime}$ to obtain a higher signal-to-noise ratio. Levels are spaced by $0.5 \mathrm{MJy} \mathrm{sr}^{-1}$ from 0.5 to $4 \mathrm{MJy} \mathrm{sr}^{-1}$. The dashed line represents the cut observed with PRONAOS, and the white section the fraction of the cut observed with the IRAM- $30 \mathrm{~m}$. The original cut direction was chosen to be perpendicular to the FIR filament (colour image on-line). The $(0,0)$ position corresponds to $\alpha_{2000}=4^{\mathrm{h}} 18^{\mathrm{m}} 50^{\mathrm{s}}$, $\delta_{2000}=+25^{\circ} 19^{\prime} 15^{\prime \prime}$.

Table 1. Telescope settings.

\begin{tabular}{ccccc}
\hline \hline Line & $\begin{array}{c}\text { Frequency } \\
(\mathrm{MHz})\end{array}$ & $\begin{array}{c}\text { Beam size } \\
\left({ }^{\prime \prime}\right)\end{array}$ & \multicolumn{2}{c}{ Sampling } \\
$(\mathrm{kHz})$ & $\mathrm{m} \mathrm{s}^{-1}$ \\
\hline $\mathrm{N}_{2} \mathrm{D}^{+}(J: 1-0)$ & $77109.616^{a}$ & 32 & 6 & 23 \\
$\mathrm{~N}_{2} \mathrm{H}^{+}(J: 1-0)$ & $93173.764^{a}$ & 27 & 10 & 32 \\
$\mathrm{C}^{18} \mathrm{O}(J: 1-0)$ & 109782.176 & 23 & 10 & 27 \\
${ }^{13} \mathrm{CO}(J: 1-0)$ & 110201.360 & 23 & 20 & 54 \\
$\mathrm{C}^{17} \mathrm{O}(J: 1-0)$ & $112358.982^{b}$ & 23 & 10 & 27 \\
$\mathrm{C}^{18} \mathrm{O}(J: 2-1)$ & 219560.358 & 12 & 40 & 54 \\
\hline
\end{tabular}

Notes. ${ }^{(a)}$ New frequency for the main $J_{\mathrm{FF}^{\prime}}: 1_{23} \rightarrow 0_{12}$ hyperfine component from Pagani et al. (2009a).

(b) $J_{\mathrm{F}}: 1_{7 / 2}-0_{5 / 2}$ transition from Klapper et al. (2003).

cases, the $\operatorname{VESPA}^{7}$ autocorrelator was used with frequency sampling varying from 6 to $20 \mathrm{kHz}$ for $3 \mathrm{~mm}$ lines and $40 \mathrm{kHz}$ for $1.3 \mathrm{~mm}$ lines (Table 1). All observations were performed in frequency switching mode. Receivers $A \& B$ were used except in July 2008, when the $1 \mathrm{~mm} 9$ pixel cameras HERA ${ }^{8} 1 \& 2$ were used in the On-the-Fly mode still using frequency switching. The $\mathrm{C}^{18} \mathrm{O}$ and ${ }^{13} \mathrm{CO}(\mathrm{J}: 1-0)$ lines were observed simultaneously by tuning the receiver halfway between the two frequencies and offsetting the VESPA autocorrelator subwindows by $\pm 210 \mathrm{MHz}$ in front of the two lines. Pointing was regularly checked and found to be stable to within $3^{\prime \prime}$.

\section{Results and analysis}

Figure 2 shows a large-scale ${ }^{13} \mathrm{CO}(\mathrm{J}: 1-0)$ map obtained with POM- ${ }^{9}$, a $2.5 \mathrm{~m}$ dish telescope with $4.5^{\prime}$ resolution and

\footnotetext{
7 VErsatile Spectroscopic and Polarimetric Analyzer, see IRAM website.

${ }^{8}$ HEterodyne Receiver Array, see IRAM website.

9 Petite Opération Millimétrique.
} 


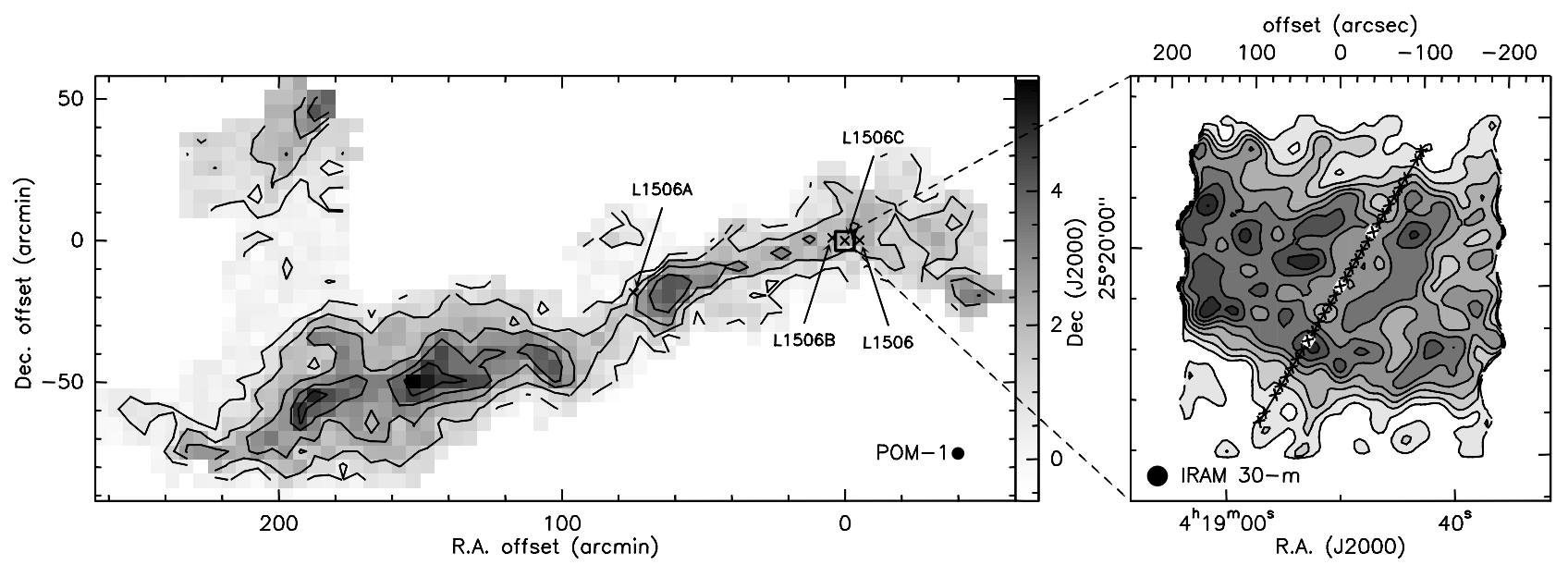

Fig. 2. Left: low resolution ${ }^{13} \mathrm{CO}(\mathrm{J}: 1-0)$ integrated intensity map of the L1506 Taurus cloud filament (original data from Nercessian et al. 1988). Contour levels are 0.5 to $4.5 \mathrm{~K} \mathrm{~km} \mathrm{~s}^{-1}$ in steps of $1 \mathrm{~K} \mathrm{~km} \mathrm{~s}^{-1}$. The POM-1 $2.5-\mathrm{m}$ dish has a $4.5^{\prime}$ resolution as shown in the bottom right corner. The square indicates the surface mapped in $\mathrm{C}^{18} \mathrm{O}(J: 2-1)$. The offsets are with respect to L1506C coordinates. Right: the smoothed $\mathrm{C}^{18} \mathrm{O}(\mathrm{J}: 2-1)$ integrated intensity map. Superimposed on the map is the strip path observed with PRONAOS (Stepnik et al. 2003) along which we observed ${ }^{13} \mathrm{CO}, \mathrm{C}^{18} \mathrm{O}$, and $\mathrm{N}_{2} \mathrm{H}^{+}$with a high signal-to-noise ratio (see Fig. 3). The crosses indicate the observed points and the 3 white boxes (superimposed on 3 crosses) the points observed also in $\mathrm{C}^{17} \mathrm{O}$ (see Fig. 5). The degraded $30-\mathrm{m}$ beam size is represented. Levels are from 0.1 to $0.7 \mathrm{~K} \mathrm{~km} \mathrm{~s}^{-1}$ in steps of $0.1 \mathrm{~K} \mathrm{~km} \mathrm{~s}^{-1}$. The $(0,0)$ position corresponds to $\alpha_{2000}=4^{\mathrm{h}} 18^{\mathrm{m}} 50^{\mathrm{s}}, \delta_{2000}=+25^{\circ} 19^{\prime} 15^{\prime \prime}$.

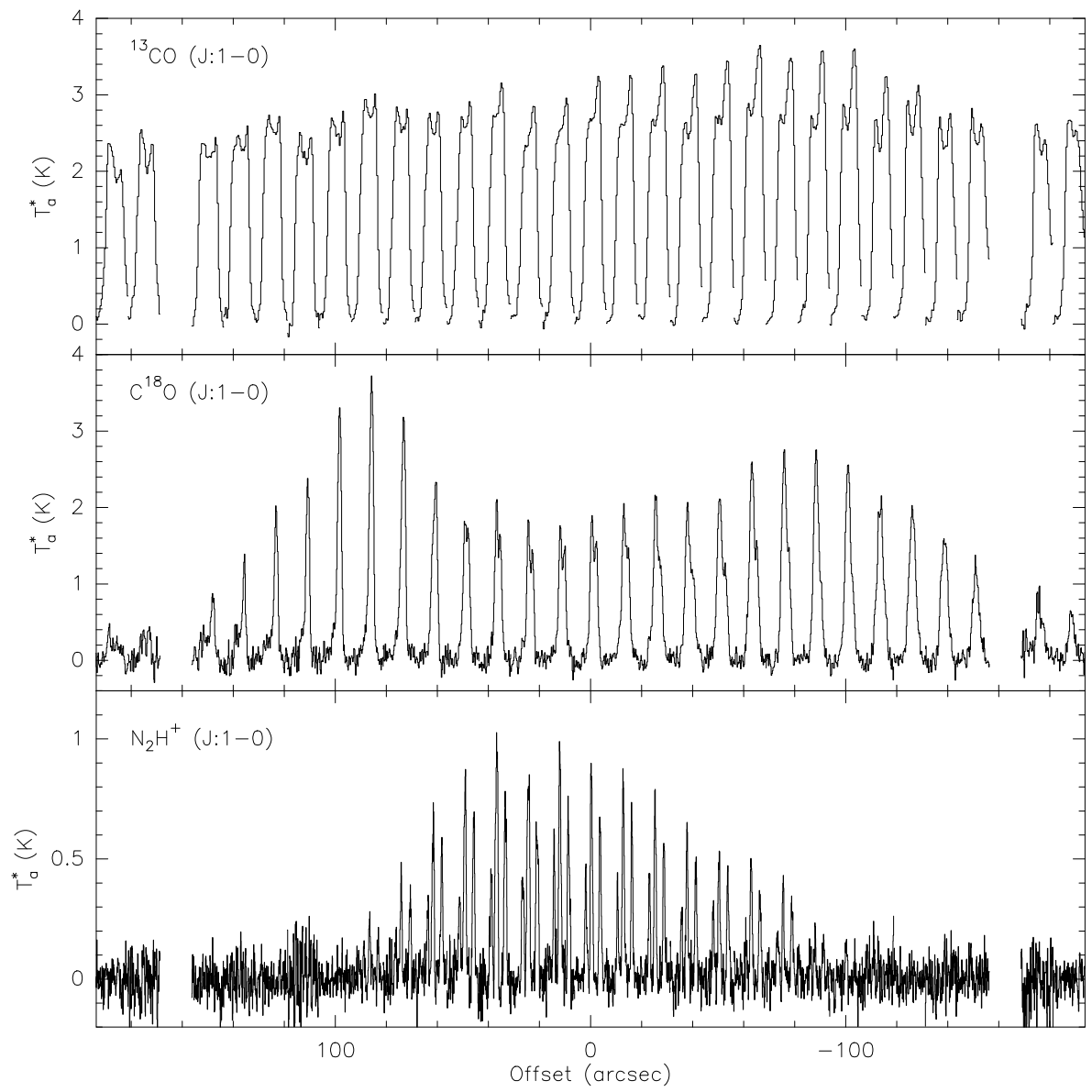

Fig. 3. Spectra taken along the PRONAOS cut (see Fig. 2). The upper row shows the ${ }^{13} \mathrm{CO}(J: 1-0)$ spectra. The middle row shows the $\mathrm{C}^{18} \mathrm{O}(J: 1-0)$ spectra. The lower row shows the $\mathrm{N}_{2} \mathrm{H}^{+}(J: 1-0)$ spectra. The velocity scale is 6.5 to $8 \mathrm{~km} \mathrm{~s}^{-1}$ for ${ }^{13} \mathrm{CO}$ and $\mathrm{C}^{18} \mathrm{O}$, and 5.5 to $9 \mathrm{~km} \mathrm{~s}^{-1}$ for $\mathrm{N}_{2} \mathrm{H}^{+}$. Offsets increase from North-West to South-East.
$100 \mathrm{kHz}$ filters $-0.27 \mathrm{~km} \mathrm{~s}^{-1}$ - that was in service at Bordeaux Observatory. The original data were taken from Nercessian et al. (1988) and the region of interest is delineated by a square. This map traces the large-scale envelope of the cloud. On the right-hand side, the $\mathrm{C}^{18} \mathrm{O}(\mathrm{J}: 2-1)$ integrated intensity map obtained on-the-fly with the $30-\mathrm{m}$ over L1506C is shown. We degraded the resolution to $24^{\prime \prime}$ to increase the signal-to-noise ratio. A large hole in emission is visible in the centre of the map which is caused by $\mathrm{CO}$ depletion, as now well established. The PRONAOS strip centred on the $100 \mu \mathrm{m}$ emission peak traverses the most depleted part of the filament. This depletion is also remarkable in terms of spectrum peak temperature (Fig. 3) or integrated intensity (Fig. 4) along the PRONAOS strip. In these figures, the decrease in $\mathrm{C}^{18} \mathrm{O}$ intensity is correlated with the 


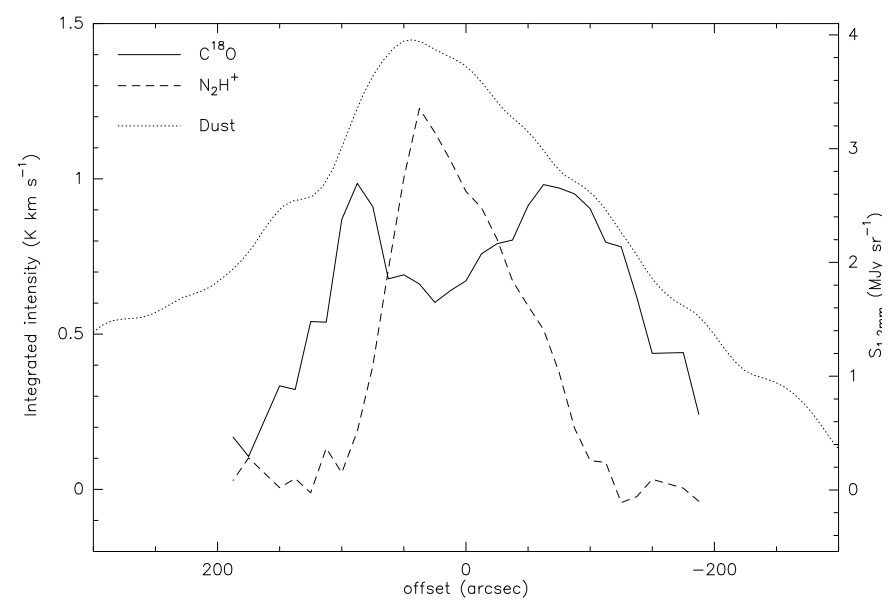

Fig. 4. Integrated intensity of $\mathrm{C}^{18} \mathrm{O}$ and $\mathrm{N}_{2} \mathrm{H}^{+}(J: 1-0)$ compared to the dust emission as measured by MAMBO along the PRONAOS cut. MAMBO data are smoothed to $40^{\prime \prime}$ here. The core is clearly not symmetrical and $\mathrm{C}^{18} \mathrm{O}$ depletion is obvious.

appearance of $\mathrm{N}_{2} \mathrm{H}^{+}$, another sure sign of $\mathrm{CO}$ depletion as $\mathrm{CO}$ readily destroys $\mathrm{N}_{2} \mathrm{H}^{+}$(Bergin \& Langer 1997; Caselli 2002; Pagani et al. 2005). To verify that the $\mathrm{C}^{18} \mathrm{O}$ lines do not decrease for opacity reasons in the centre of the cloud, we observed 3 positions in $\mathrm{C}^{17} \mathrm{O}(\mathrm{J}: 1-0)$. We chose two positions close to the $\mathrm{C}^{18} \mathrm{O}$ peaks on each side of the strip $\left( \pm 75^{\prime \prime}\right)$ and one in the middle, close to the local minimum of the $\mathrm{C}^{18} \mathrm{O}$ emission (Fig. 5). These 3 positions are indicated in Fig. 2 by 3 small white boxes superimposed on 3 of the crosses along the PRONAOS strip. Several interesting features are visible:

1. The $(0,0)$ and $\left(+75^{\prime \prime}, 0\right)$ offset positions (along the cut) display extremely narrow $\mathrm{C}^{17} \mathrm{O}$ and $\mathrm{C}^{18} \mathrm{O}$ lines $(F W H M=0.16$ to $0.26 \mathrm{~km} \mathrm{~s}^{-1}$, where $F W H M$ represents full width half maximum).

2. The existence of a velocity gradient across the strip, which possibly traces rotation in the $\mathrm{C}^{18} \mathrm{O}$ data.

3. The existence of another velocity gradient for ${ }^{13} \mathrm{CO}$ of opposite direction.

4. The $\mathrm{C}^{18} \mathrm{O}(J: 1-0)$ splitting towards the reference position, which is not very visible in the $(J: 2-1)$ line but is clearly so in the $\mathrm{C}^{17} \mathrm{O}(\mathrm{J}: 1-0)$ line, as the dotted lines indicate in Fig. 5.

Since most of the properties of this cloud change gradually with radius, it is difficult to differentiate between the core and the envelope. One parameter that changes abruptly however is the velocity field. We therefore define the envelope as the region where ${ }^{13} \mathrm{CO}$ exhibits a different velocity field from the other species, while $\mathrm{C}^{18} \mathrm{O}$ and $\mathrm{N}_{2} \mathrm{H}^{+}$both trace the core, the outer, undepleted part the former and the inner depleted one the latter.

As far as we know, these $\mathrm{CO}$ narrow lines are the narrowest $\mathrm{C}^{18} \mathrm{O}$ and $\mathrm{C}^{17} \mathrm{O}$ lines reported so far, being comparable to the very narrow features seen in $\mathrm{NH}_{3}$ and $\mathrm{N}_{2} \mathrm{H}^{+}$in sources such as L183 (Pagani et al. 2007). The main difference is that these $\mathrm{C}^{18} \mathrm{O}$ and $\mathrm{C}^{17} \mathrm{O}$ lines are emitted outside the depleted core, in a region where turbulence is usually still high. The lines are narrow enough for the $\mathrm{C}^{17} \mathrm{O} J_{\mathrm{F}}: 1_{7 / 2}-0_{5 / 2}$ and $J_{\mathrm{F}}: 1_{3 / 2}-0_{5 / 2}$ hyperfine components, which are usually blended, to be completely separated here.

Figure 6 confirms the extraordinary narrowness of the lines towards the centre of the cloud. These $\mathrm{C}^{18} \mathrm{O}$ spectra were taken at $\left(0,-25^{\prime \prime}\right)$ in the frame of the PRONAOS cut, which is

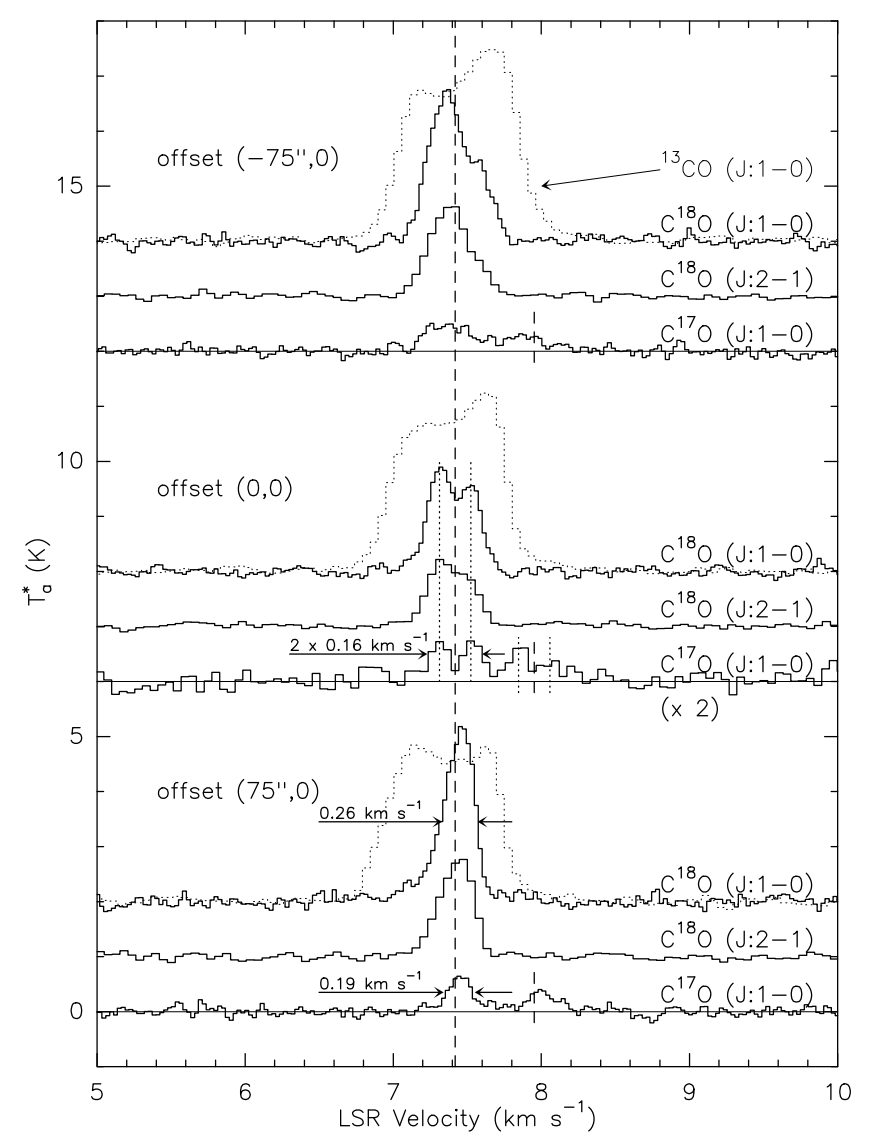

Fig. 5. Spectra of ${ }^{13} \mathrm{CO}$ (dotted lines), $\mathrm{C}^{17} \mathrm{O}$, and $\mathrm{C}^{18} \mathrm{O}$ taken at 3 different positions along the PRONAOS strip: in the middle and close to each side peak $\mathrm{C}^{18} \mathrm{O}$ emission. The full width at half maximum is indicated for some remarkably narrow lines. Only the $\mathrm{C}^{17} \mathrm{O} J_{\mathrm{F}}: 1_{7 / 2}-0_{5 / 2}$ and $J_{\mathrm{F}}: 1_{3 / 2}-0_{5 / 2}$ are displayed. The dashed line marks the systemic velocity (with an offset of $+0.534 \mathrm{~km} \mathrm{~s}^{-1}$ for the $J_{\mathrm{F}}: 1_{3 / 2}-0_{5 / 2} \mathrm{C}^{17} \mathrm{O}$ transition), while the dotted lines for the $\mathrm{C}^{17} \mathrm{O}$ transition indicate the symmetrical velocity displacement of each $\mathrm{C}^{17} \mathrm{O}$ hyperfine component in the $(0,0)$ direction (see text). Lines are artificially offset vertically and the central offset $\mathrm{C}^{17} \mathrm{O}$ line is multiplied by a factor of 2 . The $\mathrm{C}^{18} \mathrm{O}$ $(J: 2-1)$ lines are shown at their original $11^{\prime \prime}$ resolution.

$\left(-21.6^{\prime \prime},-12.6^{\prime \prime}\right)$ in the Equatorial J2000 frame. The $(J: 1-0)$ spectrum clearly contains two components, which we fitted with 2 independent Gaussians. We also fitted the $(J: 2-1)$ spectrum with two unconstrained Gaussians. The velocity of each of the two components agree for both lines within the uncertainty of the fit (a difference of $10 \mathrm{~m} \mathrm{~s}^{-1}$ for an uncertainty of 30 and $40 \mathrm{~m} \mathrm{~s}^{-1}$ for the left and right components, respectively). As for $\mathrm{C}^{17} \mathrm{O}$ in Fig. 5, the splitting of the two components is $\sim 200 \mathrm{~m} \mathrm{~s}^{-1}$ and the width of the individual components is 150 (right) and 180 (left) $\mathrm{m} \mathrm{s}^{-1}$. For a standard gas kinetic temperature of $10 \mathrm{~K}$, for which the optically thin thermal linewidth of $\mathrm{CO}$ is $\sim 0.12 \mathrm{~km} \mathrm{~s}^{-1}$, this constrains the turbulent width contribution to the linewidth to be $\$ 90 \mathrm{~m} \mathrm{~s}^{-1}$.

If the $\mathrm{C}^{18} \mathrm{O}$ and $\mathrm{C}^{17} \mathrm{O}$ splitting was caused by selfabsorption, the exact reverse would be true, the $\mathrm{C}^{18} \mathrm{O}(\mathrm{J}: 2-1)$ line would have contained the deepest self-absorption feature and normally no such feature would have been seen in $\mathrm{C}^{17} \mathrm{O}$ since, due to its low abundance and to its hyperfine structure, it is very difficult to make it optically thick. These symmetric features cannot be caused by rotational Döppler shifting because for a narrow beam directed at the rotational axis, most of the material picked up by the beam moves at right angles to the line of 


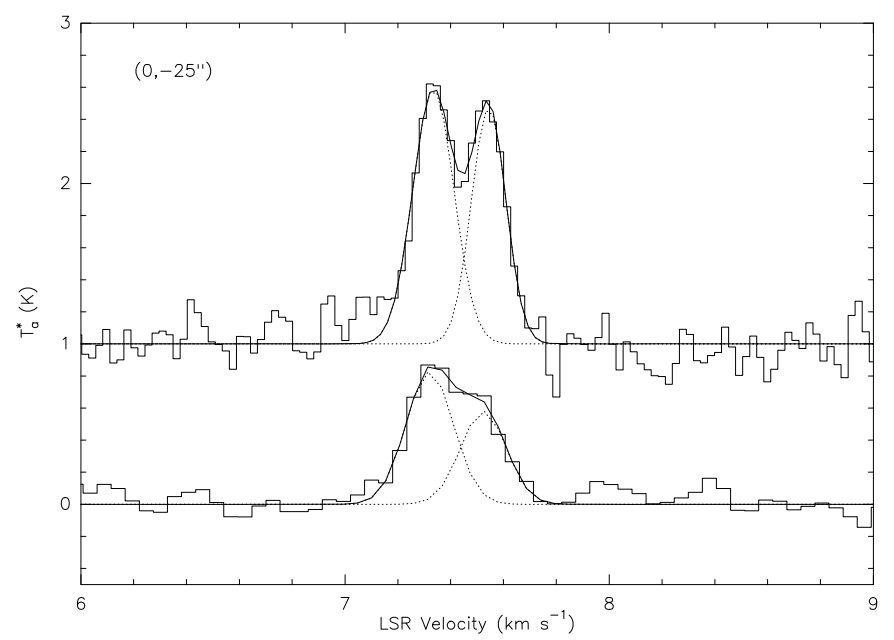

Fig. 6. $\mathrm{C}^{18} \mathrm{O}(\mathrm{J}: 1-0)($ top $)$ and $(J: 2-1)$ (bottom) spectra (histogram plot) taken $25^{\prime \prime}$ away from the PRONAOS cut. The total Gaussian fit (full line) for both transitions is shown as well as individual components. The components are separated by $200 \mathrm{~m} \mathrm{~s}^{-1}$ and their width is 150 (right) and $180($ left $) \mathrm{m} \mathrm{s}^{-1}$.

sight with therefore no contribution to the velocity component along it. Only material very close to the rotational axis would contribute to the Döppler shifted emission. Since the inner core is depleted in $\mathrm{CO}$, this contribution near the axis is not possible and therefore rotation cannot explain this splitting. To explain this double peak feature, the only possibility left is that the $\mathrm{CO}$ envelope is radially moving. The direction of movement (expansion or contraction) can be inferred from the asymmetry of the $\mathrm{C}^{18} \mathrm{O}(\mathrm{J}: 1-0)$ and $(J: 2-1)$ lines. Here, these $\mathrm{C}^{18} \mathrm{O}$ lines are stronger in the blue component than in the red component. As we confirm using the Monte Carlo model (Sect. 4), this can only be explained by contraction and not expansion despite the absence of heavy self-absorption, which is usually invoked to justify blue peaked profiles (Myers et al. 1996). Since the lines are almost independent because of their narrowness, the blue and red line contributions are each emitted from only one side of the cloud - front or back depending on the movement, expansion or contraction - and have no or very little interaction. The line formed in the rear part of the core originates in the (backside) low density envelope and obtains contribution from a material that becomes denser as the photons travel towards the observer, until they reach the depleted zone and then cross the remaining cloud without interaction. For a constant kinetic temperature, this means that the excitation temperature of the contributing material increases (or remains constant if thermalized) towards the observer and the successive layers add their contribution to the emergent signal. In contrast, the line formed on the near side of the cloud originates in the highest density and excitation temperature layer, just outside the depleted zone and obtains contributions from increasingly less dense material with therefore decreasing excitation temperature. When the excitation temperature decreases, a net absorption of photons can occur if opacity is not null. The $\mathrm{C}^{18} \mathrm{O}$ lines are not really optically thin (opacities are in the range of 0.1 to 0.5 ) and this is enough to slightly differentiate between the line that originates in the rear part of the cloud, which experiences no attenuation after leaving the highest excitation temperature layer and the line which comes from the front part and experienced a small absorption due to the outer, less excited layers. The effect is clearly visible towards the $(0,0)$ position: the $\mathrm{C}^{18} \mathrm{O}(\mathrm{J}: 1-0)$ line is $20 \%$ stronger on the blue side ( 1.81 and $1.48 \mathrm{~K}$ peak temperature for the blue and red components, a difference greater than $10 \sigma$, with $\sigma=27 \mathrm{mK})$, and the $\mathrm{C}^{18} \mathrm{O}(\mathrm{J}: 2-1)$ line has lost its red peak because of higher opacity and higher sensitivity to density conditions (Fig. 5). The red component is therefore that emitted from the front part of the cloud, the blue component from the rear part and this indicates that the cloud is contracting rather than expanding ${ }^{10}$. The amount of contraction of the $\mathrm{C}^{18} \mathrm{O}$ core is indicated by the two dotted lines in Fig. 5 , namely $\approx \pm 100 \mathrm{~m} \mathrm{~s}^{-1}$. Therefore, the $\mathrm{C}^{18} \mathrm{O}$ outer core both rotates (from the $\left( \pm 75^{\prime \prime}, 0\right)$ symmetrical velocity offsets seen in Fig. 5 as mentioned above) and contracts. To our knowledge, this is the first time that core contraction is observed based on $\mathrm{C}^{18} \mathrm{O}$ and $\mathrm{C}^{17} \mathrm{O}$ lines.

The $\mathrm{N}_{2} \mathrm{H}^{+}$hyperfine structure lines do not have such a high signal-to-noise ratio and do not show structured profiles. However, their displacement in velocity along the cut is similar to that of $\mathrm{C}^{18} \mathrm{O}$ as we can see in Fig. 7.

The ${ }^{13} \mathrm{CO}(J: 1-0)$ line, which was observed simultaneously with the $\mathrm{C}^{18} \mathrm{O}(J: 1-0)$ line, has a very different behaviour (Figs. 3, 5 and 7). Its peak intensity is redshifted and its velocity gradient along the cut is opposite to that of the $\mathrm{C}^{18} \mathrm{O}$ line. From the large-scale ${ }^{13} \mathrm{CO}$ map obtained with POM-1, we know that the L1506 filament has a width of $\sim 40^{\prime}$ in the region around L1506C (Fig. 2). This is $\sim 6$ times larger than the $\mathrm{C}^{18} \mathrm{O}$ extent. As we do not know how the ${ }^{13} \mathrm{CO}$ velocity behaves beyond these central $6^{\prime}$, we cannot claim that this velocity drift is the signature of a rotation of the large-scale filament but it definitely indicates that it is opposite to the $\mathrm{C}^{18} \mathrm{O}$ rotation (Figs. 5 and 7). Similarly, the red peak in the ${ }^{13} \mathrm{CO}$ velocity profile is probably a sign of gas expansion (Figs. 3 and 5), while $\mathrm{C}^{18} \mathrm{O}$ traces a contraction. Therefore, the extended envelope has a dynamical behaviour that is the opposite of that of the core.

Figure 4 shows the asymmetry of the cloud in both dust and gas. It is remarkable that the $\mathrm{C}^{18} \mathrm{O}$ lines on each side of the cut have the same maximum integrated intensity despite a variation of $25 \%$ in peak intensity. It indicates that the quantity of gas is comparable and that the main reason for the intensity change is the line width difference. We also note that the $\mathrm{N}_{2} \mathrm{H}^{+}$integrated intensity profile is strongly asymmetric and varies abruptly at the top, two features that we cannot reproduce with our standard one-dimensional (1D) model (see Sect. 4). Its peak position is close to but not coincident with the dust peak position.

We observed the position with the second strongest $\mathrm{N}_{2} \mathrm{H}^{+}$ integrated intensity (offset $+25^{\prime \prime}$ along the PRONAOS strip) in $\mathrm{N}_{2} \mathrm{D}^{+}(J: 1-0)$ to check the level of deuteration in the inner core (the strongest peak - offset $+37.5^{\prime \prime}$ - was identified afterwards). We found only a very weak signal (Fig. 8), the main hyperfine component being only $70 \mathrm{mK}$. A hyperfine fit was performed using the CLASS fitting routine MINIMIZE with the HFS option ${ }^{11}$.

10 There exists the alternative possibility that the blue peak is stronger than the red peak because of a difference in column densities between front and rear parts of the cloud. If this were the case in such a manner that it would hide the cloud expansion, the difference in column density between the two components would be $\sim 40 \%$ in the optically thin limit (and even more with growing opacity). Indeed, since the intensity difference is about $20 \%$ towards the $(0,0)$ position in $\mathrm{C}^{18} \mathrm{O}(\mathrm{J}: 1-0)$, it would have to be compensated twice to go from a stronger red component (due to expansion combined with differential excitation) to a weaker red component (as observed) and the effect would be more pronounced for the $(J: 2-1)$ component. This possibility seems therefore quite unlikely and even if the two sides of the cloud have different column densities, contraction remains the most probable explanation.

11 http://wWw.iram.fr/IRAMFR/GILDAS/ 


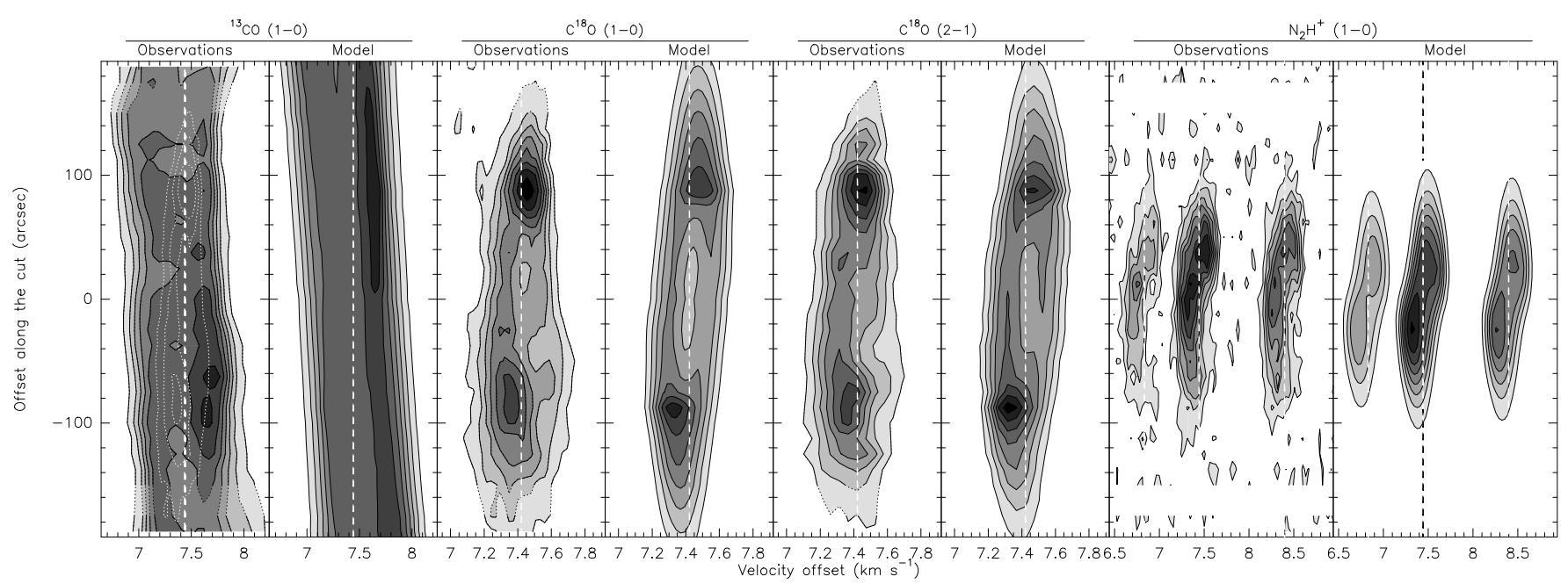

Fig. 7. Position-velocity plots along the PRONAOS cut. For each line, we show the observations on the left and the model on the right with the same contour levels. For $\mathrm{N}_{2} \mathrm{H}^{+}$, we reproduce only the 3 inner hyperfine components. The dashed line indicates the systemic velocity of the cloud. For ${ }^{13} \mathrm{CO}$ and $\mathrm{C}^{18} \mathrm{O}$ observations, the dotted contours delineate interpolated data (data is missing for offsets $\pm 162.5^{\prime \prime}$ ). Superimposed to the ${ }^{13} \mathrm{CO}$ observations, dotted contour levels from the $\mathrm{C}^{18} \mathrm{O}(\mathrm{J}: 1-0)$ observation plot are repeated. Only levels $1-3 \mathrm{~K}$ have been drawn for clarity.

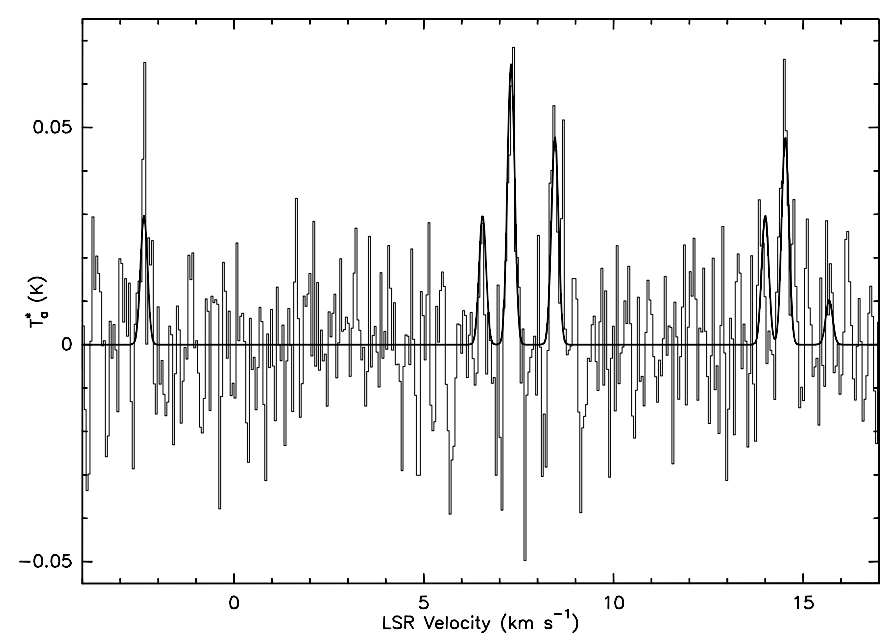

Fig. 8. Spectrum of $\mathrm{N}_{2} \mathrm{D}^{+}$taken at offset $+25^{\prime \prime}$ along the PRONAOS strip. The hyperfine fit is superposed to the data. The data is Hanning smoothed once. The sampling is $50 \mathrm{~m} \mathrm{~s}^{-1}$ and the rms is $13 \mathrm{mK}$.

\section{Models}

\subsection{Density profile}

The density radial distribution was derived from the MAMBO map emission using a Wiener linear inversion method as described by Dupac \& Giard (2002). Considering the elongated distribution (along the RA axis), we modelled the filament with a cylindrical geometry approximation, its axis being in the plane of the sky and perpendicular to the PRONAOS strip, as in Stepnik et al. (2003). We also accounted for a temperature inward decrease from $15 \mathrm{~K}$ to $8 \mathrm{~K}$. This range was inferred from the $A_{\mathrm{v}}$ profile of the filament (Stepnik et al. 2003) combined with the predictions of either the Zucconi et al. (2001) or Bernard et al. (1992) dust temperature models in cold cores. We discretized the cylinder into iso-density and iso-temperature rings, and defined the array A to be

$N_{\mathrm{H} 2}(j)=A(i, j) \times n_{\mathrm{H} 2}(i)+b$ or

$I_{\lambda}^{j}=\sum_{i} A(i, j) \times B_{\lambda}\left(T_{i}\right) \times \kappa_{1200 \mu \mathrm{m}} \times m_{\mathrm{H}} \times \mu \times n_{\mathrm{H} 2}(i)+b$,

where $(i, j)$ are, respectively, the indices of the rings and the lines of sight, $N_{\mathrm{H} 2}$ the column density, $n_{\mathrm{H} 2}(i)$ the gas density in the ring $i, I_{\lambda}^{j}$ the observed intensity along the line of sight $j, b$ is the noise level, $B_{\lambda}\left(T_{i}\right)$ is the black-body function evaluated for the temperature $T$ of the ring $i, m_{\mathrm{H}}$ is the proton mass, $\mu=2.33$ is the mean molecular weight of interstellar material in molecular clouds and $\kappa_{1200 \mu \mathrm{m}}=8 \times 10^{-3} \mathrm{~cm}^{-2} \mathrm{~g}^{-1}$ is the mass absorption coefficient which is assumed to be constant in the core and derived from the dust aggregates emissivity model by Ossenkopf \& Henning (1994). The local density $n_{\mathrm{H} 2}$ in each ring $(i)$ is assumed to be $n_{\mathrm{H} 2}=W \times I_{\lambda}$, where we adopt the optimized inversion matrix

$W=\left({ }^{t} A^{\prime} \times A^{\prime}\right)^{-1} \times{ }^{t} A^{\prime}$,

(cf. Dupac \& Giard 2002), where

$A^{\prime}=\kappa_{1200 \mu \mathrm{m}} \times m_{\mathrm{H}} \times A(i, j) \times B_{\lambda}\left(T_{i}\right)$.

The most successful optimization was obtained using a ring thickness value of $50^{\prime \prime}$, the result leading to an inner density of $2.4 \times 10^{4} \mathrm{~cm}^{-3}$ (averaged over a $50^{\prime \prime}$ radius core).

\subsection{Line modeling}

The density profile derived from the dust data was used as a first estimate in our 1D line radiative transfer models but we allowed the profile to change because the raw dust map is noisy and its final resolution in the inversion method is only $50^{\prime \prime}$ compared to the $\mathrm{C}^{18} \mathrm{O}(\mathrm{J}: 1-0) 24^{\prime \prime}$ and $(\mathrm{J}: 2-1) 12^{\prime \prime}$ and because the dust properties are not strongly constrained and can vary, therefore causing a typical uncertainty of a factor of 2 in the derived density.

For all three species, ${ }^{13} \mathrm{CO}, \mathrm{C}^{18} \mathrm{O}$, and $\mathrm{N}_{2} \mathrm{H}^{+}$, we fit a nonlocal thermodynamic equilibrium radiative transfer Monte Carlo model and attempt to reproduce the spectra (Figs. 9-12) and the velocity-position diagram (Fig. 7). The 1D Monte Carlo 

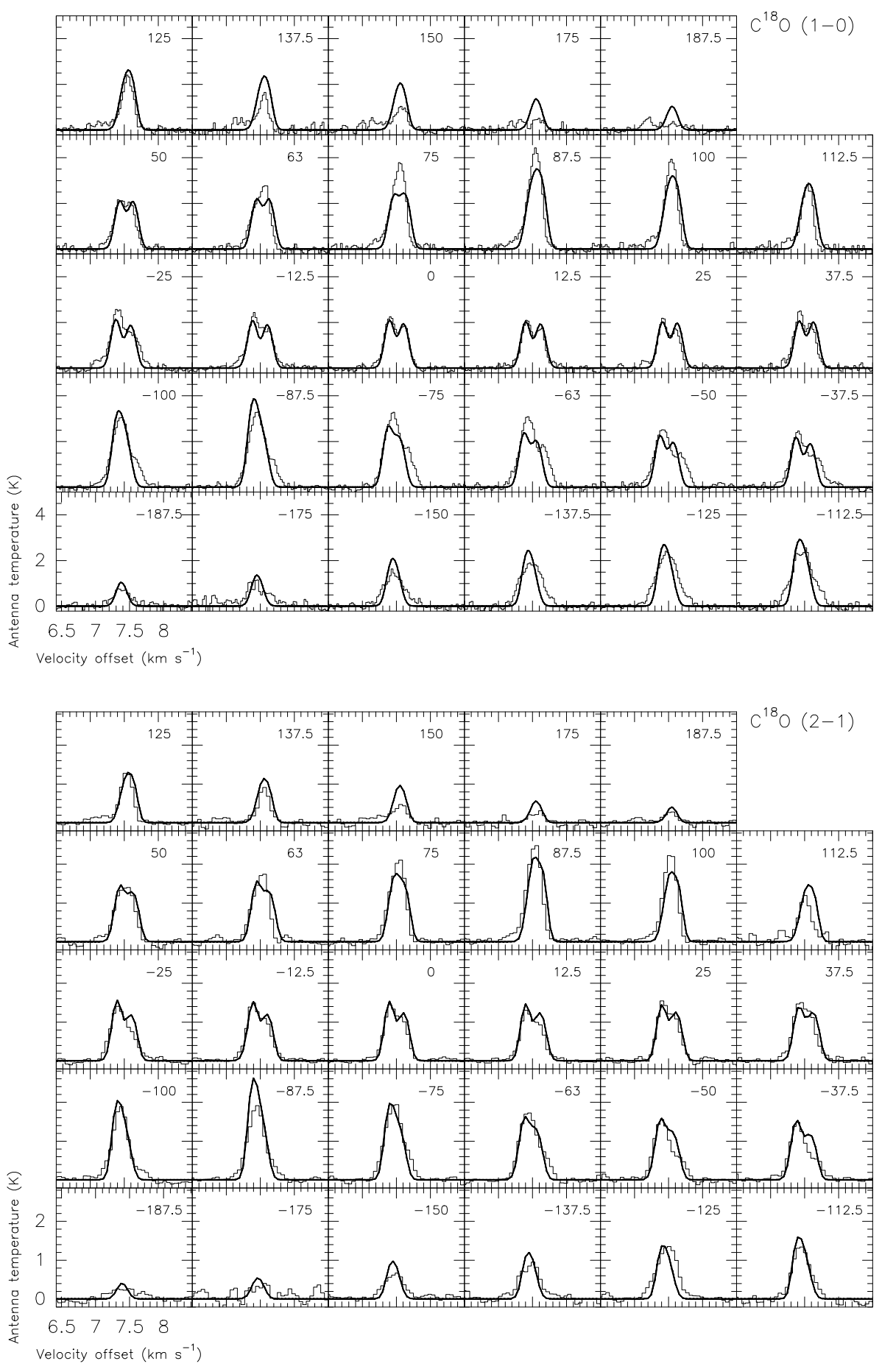

Fig. 9. Fit of the individual $\mathrm{C}^{18} \mathrm{O}(J: 1-0)$ spectra along the PRONAOS cut. The histogram plot represents the data, the thick continuous curve, the model. A FWHM turbulent velocity of $0.11 \mathrm{~km} \mathrm{~s}^{-1}$ was used throughout the cut, which explains why the model lines are larger than the observations for the positive offsets. Offsets along the PRONAOS cut in arcseconds are given in the upper right corner of each spectrum.
Fig. 10. Same as Fig. 9 for $\mathrm{C}^{18} \mathrm{O}(J: 2-1)$ spectra. model was originally developed by Bernes (1979), and incorporates both microscopic turbulence and a radial velocity field. It was subsequently modified to include rotation (Pagani \& Breart de Boisanger 1996) and a variant was developed to treat the hyperfine structure of $\mathrm{N}_{2} \mathrm{H}^{+}$(Pagani et al. 2007). The 1D cloud parameters are shown in Table 2 and the species abundances are traced as a function of radius in Fig. 13. Here the model is spherical with a radius equal to the dust cylinder radius and a rotation axis similar to the cylinder axis of the dust model, i.e., assumed to be aligned with the embedding filament elongation and therefore perpendicular to the PRONAOS strip. The model data to be compared with the observations are taken at the equator that is considered to be a good approximation of a cylinder slice, as already discussed in Pagani et al. (2007) for a similar case. For the ${ }^{13} \mathrm{CO}$ emission, since our high spatial and velocity resolution strip only extends across $6^{\prime}$ and because the ${ }^{13} \mathrm{CO}$ line is optically thick and far more extended, it is difficult to develop a simple 1D model to reproduce and constrain the 30$\mathrm{m}{ }^{13} \mathrm{CO}$ observations (the POM- 1 data are both too coarse and noisy for this purpose). More generally, the cloud is not symmetrical and therefore our 1D Monte Carlo model cannot reproduce the various differences between positive and negative offsets. However, we attempted to fit globally the observations and overall to use a similar cloud description for the $\mathrm{N}_{2} \mathrm{H}^{+}$and $\mathrm{C}^{18} \mathrm{O}$ line models. Though the ${ }^{13} \mathrm{CO}$ data are too difficult to reproduce with the 1D Monte Carlo code, we attempted to fit them as well, 


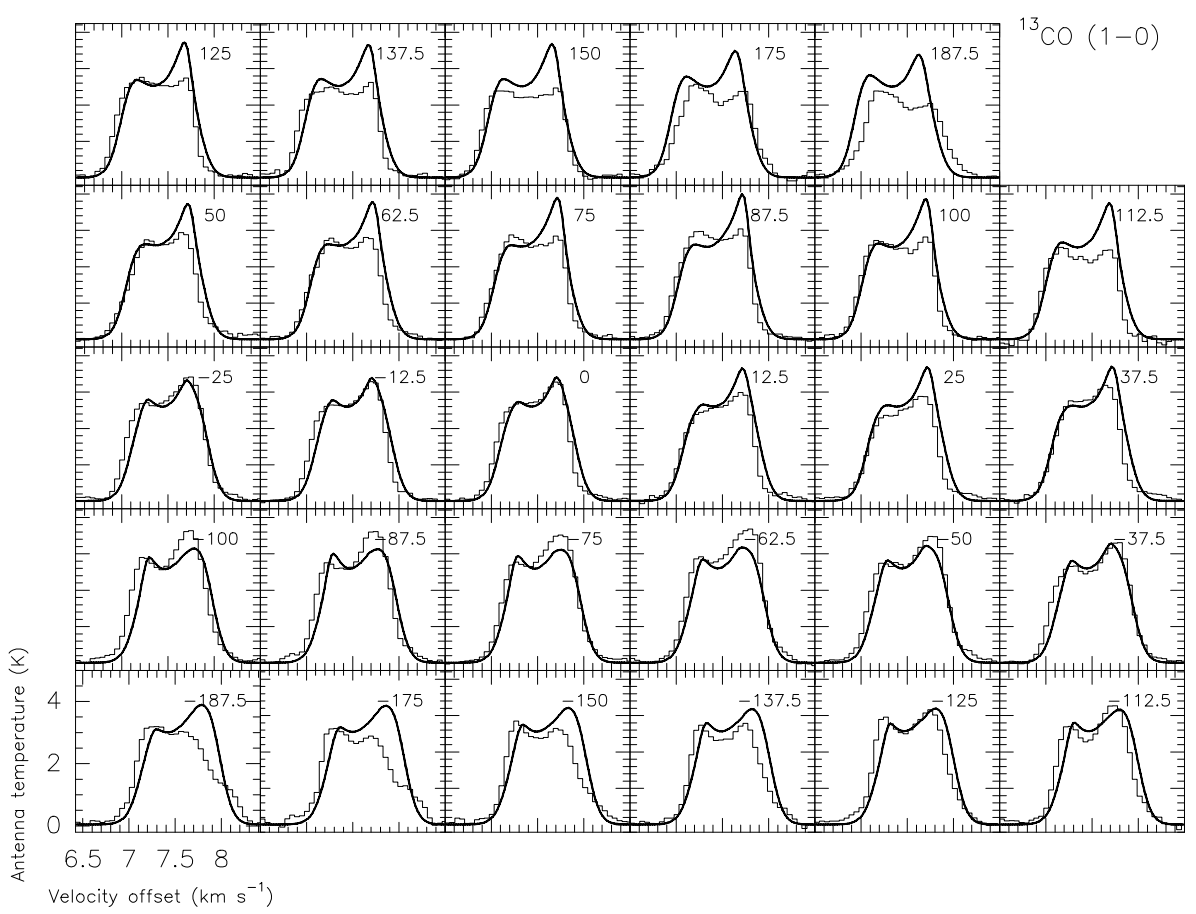

Fig. 11. Fit of the individual ${ }^{13} \mathrm{CO}(\mathrm{J}: 1-0)$ spectra along the PRONAOS cut. The histogram plot represents the data, the thick continuous curve, the model.

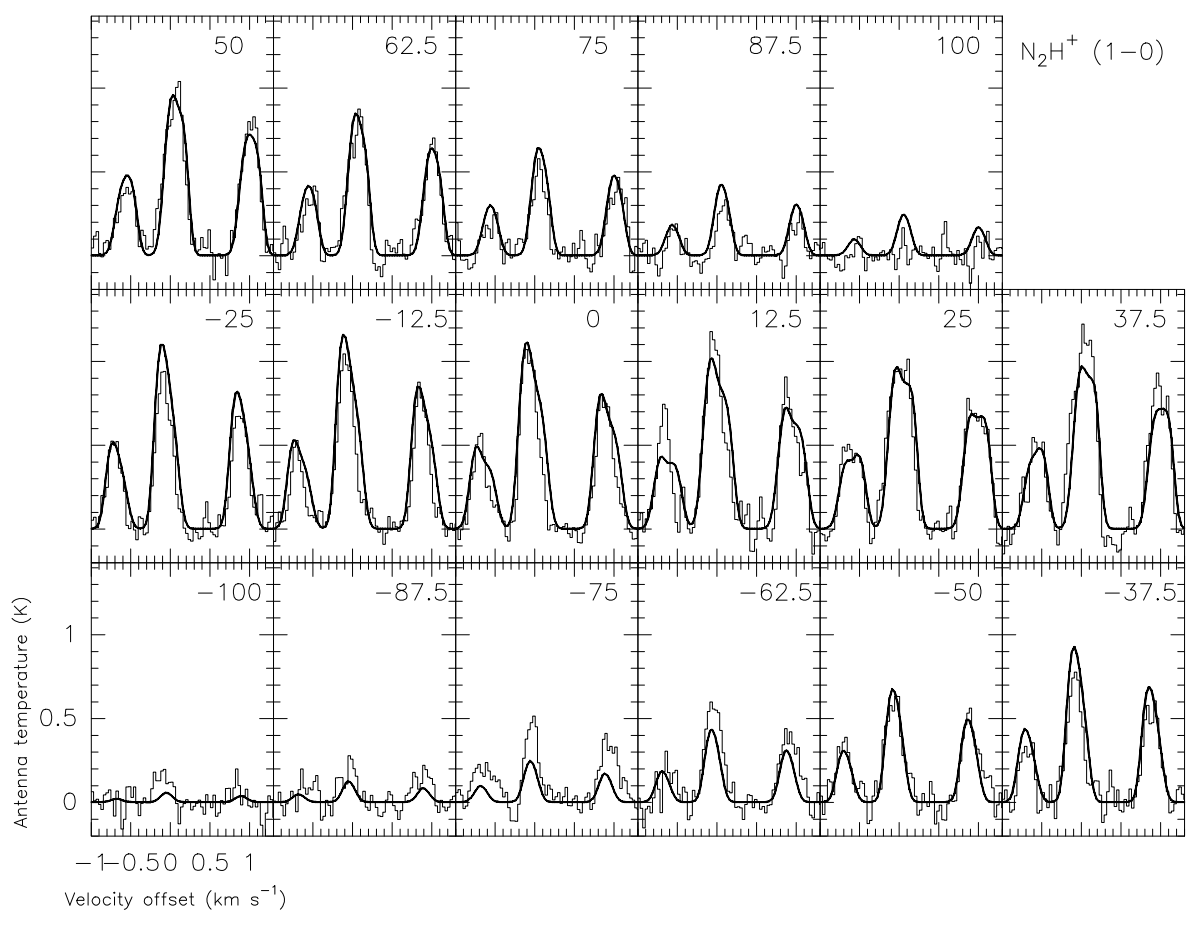

Fig. 12. Fit of the individual $\mathrm{N}_{2} \mathrm{H}^{+}(J: 1-0)$ spectra along the PRONAOS cut. The histogram plot represents the data, the thick continuous curve, the model. Only the 3 central hyperfine structure components are displayed. A turbulent velocity of $0.068 \mathrm{~km} \mathrm{~s}^{-1}$ has been used. at least to obtain some estimate of the physical conditions in the outer parts to help explain the observations. From the $\mathrm{C}^{18} \mathrm{O}$ and $\mathrm{N}_{2} \mathrm{H}^{+}$data, we find that the cloud rotational axis could also be slightly offset along the PRONAOS cut (about $+12^{\prime \prime}$ ) with respect to our reference position (which was defined somewhat arbitrarily). The peak $\mathrm{N}_{2} \mathrm{H}^{+}$integrated intensity is even farther away from the reference position $\left(+37.5^{\prime \prime}\right)$ but assuming the rotational axis to be this far does not fit with the overall velocity profile. Despite the cloud extension on the positive offset side being smaller than on the negative offset side, the fit is relatively good, especially towards the centre of the cloud. As mentioned above, we attempted to adjust the density profile finding a solution that is close to the formula of Tafalla et al. (2002) for the $\mathrm{N}_{2} \mathrm{H}^{+}-\mathrm{C}^{18} \mathrm{O}$ region

$n\left(\mathrm{H}_{2}\right)=\frac{n_{0}\left(\mathrm{H}_{2}\right)}{1+\left(\frac{r}{r_{0}}\right)^{\alpha}} \mathrm{cm}^{-3}$

where $n_{0}\left(\mathrm{H}_{2}\right)=5 \times 10^{4} \mathrm{~cm}^{-3}, r_{0}=1 \times 10^{4} \mathrm{AU}$, and $\alpha=2.5$. This profile provides a peak dust emission that is comparable to that inferred from the MAMBO observation, and the average density of $\sim 4 \times 10^{4} \mathrm{~cm}^{-3}$ in a $50^{\prime \prime}$ radius is within a factor of 2 of the peak density derived from the MAMBO observations. Apart from the dust properties that are not strongly constrained, 
Table 2. The Monte Carlo cloud model used to fit the spectra (Figs. 7 and 9-12).

\begin{tabular}{|c|c|c|c|c|c|c|c|c|c|}
\hline \multicolumn{2}{|c|}{ Shell radius } & \multirow{2}{*}{$\begin{array}{l}\text { Density } \\
\left(\mathrm{cm}^{-3}\right)\end{array}$} & \multirow{2}{*}{$\begin{array}{c}\text { Temperature } \\
\text { (K) }\end{array}$} & \multirow{2}{*}{$\begin{array}{l}\text { Rad. velocity } \\
\quad\left(\mathrm{km} \mathrm{s}^{-1}\right)\end{array}$} & \multirow{2}{*}{$\begin{array}{l}\text { Rot. velocity } \\
\quad\left(\mathrm{km} \mathrm{s}^{-1}\right)\end{array}$} & \multirow{2}{*}{$\begin{array}{l}\text { Turbulence } \\
\qquad\left(\mathrm{km} \mathrm{s}^{-1}\right)\end{array}$} & \multicolumn{3}{|c|}{ Abundance $^{c}$} \\
\hline$(\mathrm{AU})$ & $\left({ }^{\prime \prime}\right)$ & & & & & & $\mathrm{N}_{2} \mathrm{H}^{+}$ & $\mathrm{C}^{18} \mathrm{O}$ & ${ }^{13} \mathrm{CO}$ \\
\hline 1740 & 12 & 50000 & 10 & 0.11 & 0.200 & $\leq 0.11 / \leq 0.068$ & $1.5(-11)$ & $\leq 5(-9)$ & $\leq 3(-8)$ \\
\hline 3480 & 25 & 47400 & 10 & 0.11 & 0.141 & $\leq 0.11 / \leq 0.068$ & $2.5(-10)$ & $\leq 5(-9)$ & $\leq 3(-8)$ \\
\hline 5220 & 37 & 41300 & 10 & 0.11 & 0.115 & $\leq 0.11 / \leq 0.068$ & $4(-10)$ & $\leq 5(-9)$ & $\leq 3(-8)$ \\
\hline 6933 & 50 & 38100 & 10 & 0.11 & 0.100 & $\leq 0.11 / \leq 0.068$ & $5(-10)$ & $\leq 5(-9)$ & $\leq 3(-8)$ \\
\hline 8666 & 62 & 33900 & 10 & 0.11 & 0.089 & $\leq 0.11 / \leq 0.068$ & $5(-10)$ & $\leq 5(-9)$ & $\leq 3(-8)$ \\
\hline 10400 & 75 & 24300 & 10 & 0.11 & 0.082 & $\leq 0.11 / \leq 0.068$ & $4(-10)$ & $\leq 5(-9)$ & $\leq 3(-8)$ \\
\hline 12133 & 87 & 18500 & 10 & 0.11 & 0.076 & $\leq 0.11 / \leq 0.068$ & $3(-10)$ & $1(-8)$ & $6(-8)$ \\
\hline 13933 & 100 & 14700 & 10 & 0.11 & 0.070 & $\leq 0.11 / \leq 0.068$ & $2(-10)$ & $1.7(-7)$ & $1(-6)$ \\
\hline 15666 & 112 & 11900 & 9 & 0.11 & 0.066 & $\leq 0.11 / \leq 0.068$ & $1(-10)$ & $1.7(-7)$ & $1(-6)$ \\
\hline 17400 & 125 & 9990 & 8 & 0.11 & 0.063 & $\leq 0.11 / \leq 0.068$ & $1(-10)$ & $1.7(-7)$ & $1(-6)$ \\
\hline 19133 & 137 & 8540 & 8 & 0.11 & 0.060 & $\leq 0.11 / \leq 0.068$ & & $1.7(-7)$ & $1(-6)$ \\
\hline 20866 & 150 & 7400 & 8 & 0.11 & 0.058 & $\leq 0.11 / \leq 0.068$ & & $1.7(-7)$ & $1(-6)$ \\
\hline 22600 & 162 & 6100 & 8 & 0.11 & 0.055 & $\leq 0.11 / \leq 0.068$ & & $1.7(-7)$ & $1(-6)$ \\
\hline 24333 & 175 & 4800 & 8 & 0.11 & 0.053 & $\leq 0.11 / \leq 0.068$ & & $1.7(-7)$ & $1(-6)$ \\
\hline 26066 & 187 & 3850 & 8 & 0.11 & 0.052 & $\leq 0.11 / \leq 0.068$ & & $1.7(-7)$ & $1(-6)$ \\
\hline 27866 & 200 & 3130 & 8 & 0.11 & 0.050 & $\leq 0.11 / \leq 0.068$ & & $1.7(-7)$ & $1(-6)$ \\
\hline 29600 & 212 & 2560 & 8 & 0.11 & 0.048 & $\leq 0.11 / \leq 0.068$ & & $1.7(-7)$ & $1(-6)$ \\
\hline 31333 & 225 & 2140 & 8 & 0 & -0.3 & 0.4 & & $\leq 5(-9)$ & $6(-6)$ \\
\hline 46666 & 335 & 1000 & 8 & -0.09 & -0.3 & 0.4 & & $\leq 5(-9)$ & $6(-6)$ \\
\hline 66666 & 479 & 500 & 10 & -0.09 & -0.3 & 0.4 & & $\leq 5(-9)$ & $6(-6)$ \\
\hline 83333 & 598 & 400 & 12 & -0.09 & -0.3 & 0.4 & & $\leq 5(-9)$ & $6(-6)$ \\
\hline 100000 & 718 & 400 & 13 & -0.09 & -0.3 & 0.4 & & $\leq 5(-9)$ & $6(-6)$ \\
\hline 126666 & 909 & 200 & 13 & -0.09 & -0.3 & 0.4 & & $\leq 1(-9)$ & $1(-8)$ \\
\hline
\end{tabular}

Notes. ${ }^{(a)}$ Positive velocity indicates inward motion (contraction); ${ }^{(b)} F W H M$ turbulence is $\leq 0.068 \mathrm{~km} \mathrm{~s}^{-1}$ for the positive offsets in the $\mathrm{C}^{18} \mathrm{O}$ model and for all offsets in the $\mathrm{N}_{2} \mathrm{H}^{+}$model and $\leq 0.11 \mathrm{~km} \mathrm{~s}^{-1}$ otherwise (for Figs. 9 and 10 only one value could be used: $0.11 \mathrm{~km} \mathrm{~s}{ }^{-1}$ has been selected); (c) relative to $\mathrm{H}_{2} \cdot 1(-10)$ means $1 \times 10^{-10}$.

an equally probable explanation of this factor of $\lesssim 2$ difference is that the $\mathrm{He}+\mathrm{N}_{2} \mathrm{H}^{+}$collisional coefficients that we presently use (Daniel et al. 2005) overestimate the true $\mathrm{H}_{2}$ density needed to collisionally excite the $\mathrm{N}_{2} \mathrm{H}^{+}$to its $J=1$ level as discussed in Pagani et al. (2007). From dust and $\mathrm{N}_{2} \mathrm{H}^{+}$measurements, we find that the density in this core is rather low, a factor of 2 to 4 below the threshold proposed by Keto \& Caselli (2008) for prestellar cores. Therefore, it is not such a core.

The $\mathrm{CO}+\mathrm{N}_{2} \mathrm{H}^{+}$model that we present here is by no means unique but represents a family of possible solutions. This family has remarkable features that are common to all solutions:

1. A peak column density of $N\left(\mathrm{H}_{2}\right) \approx 2 \times 10^{22} \mathrm{~cm}^{-2}$, which is equivalent to $A_{\mathrm{V}} \approx 20 \mathrm{mag}$ (following Bohlin et al. 1978). This is low compared to most other cases with similar strong $\mathrm{C}^{18} \mathrm{O}$ depletion but slightly higher than in L1498, which has a peak column density of $N\left(\mathrm{H}_{2}\right) \approx 1.3 \times 10^{22} \mathrm{~cm}^{-2}$ (Shirley et al. 2005) ${ }^{12}$.

2. A strong central depletion $(\geqslant 30)$ of $\mathrm{C}^{18} \mathrm{O}$.

3. $\mathrm{C}^{18} \mathrm{O}$ depletion that begins at a density between 1.5 and $2 \times 10^{4} \mathrm{~cm}^{-3}$.

4. For both $\mathrm{C}^{18} \mathrm{O}$ lines (towards the positive offsets) and the $\mathrm{N}_{2} \mathrm{H}^{+}$line (for all offsets), the linewidth is so narrow that the turbulence contribution to the width is negligible and defined to be $V_{\text {turb }}(F W H M) \leqslant 68 \mathrm{~m} \mathrm{~s}^{-1}$. To our knowledge, this has been the lowest turbulence reported for $\mathrm{C}^{18} \mathrm{O}$ lines. The line width is mostly dominated by the thermal width and the enlargement caused by macroscopic kinematics (rotation and infall).

12 In terms of the total dust amount and the peak density of L1498, note that Shirley et al. (2005) disagree with Tafalla et al. (2004). If we were to assume that the results of the latter authors be true, L1498 would be closer to a normal prestellar core than to a simple core. The discrepancy has been only partly explained by Shirley et al. (2005).

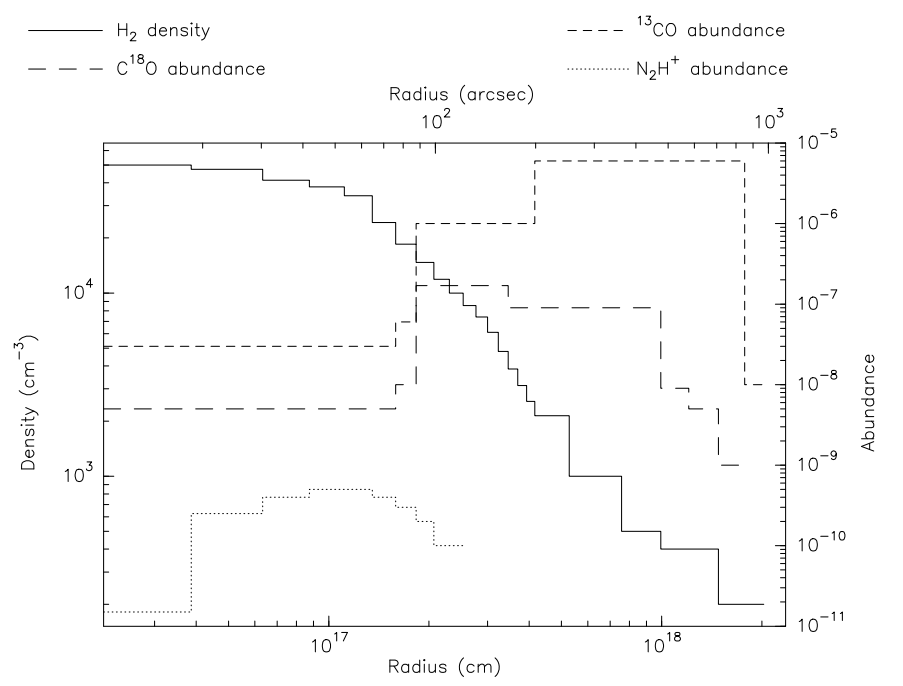

Fig. 13. $\mathrm{H}_{2}$ density and molecular abundance (relative to $\mathrm{H}_{2}$ ) as a function of radius.

5. The radial velocity is strongly constrained in the $\mathrm{C}^{18} \mathrm{O}$ envelope but not so much in the $\mathrm{N}_{2} \mathrm{H}^{+}$internal part. However, a constant infall around $0.1 \mathrm{~km} \mathrm{~s}^{-1}$ is compatible with the data. No infall is not.

6. The rotational velocity decreases with increasing radius. Solid rotation is therefore excluded and velocity dependences of the type $V \propto r^{-\alpha}$ with $\alpha \approx 0.5$ provide good solutions.

7. The temperature pattern is indicative of a slightly higher temperature in the $\mathrm{N}_{2} \mathrm{H}^{+}$inner core $(\sim 10 \mathrm{~K})$ than in the $\mathrm{C}^{18} \mathrm{O}$ outer core $(\sim 8 \mathrm{~K})$. If correct this would resemble to the B68 case (Bergin et al. 2006). 
The $\mathrm{C}^{18} \mathrm{O}$ temperature is determined by the necessity to reproduce both the $(J: 1-0)$ and $(J: 2-1)$ lines with a relatively tightly constrained dust profile, whereas the $\mathrm{N}_{2} \mathrm{H}^{+}$temperature is the minimum we could define while keeping the needed density as low as possible. Despite the observation of a single transition for $\mathrm{N}_{2} \mathrm{H}^{+}$, we are constrained by the hyperfine structure (see Pagani et al. 2007). However, this temperature difference could possibly prove to be inaccurate if the actual $\mathrm{H}_{2}-\mathrm{N}_{2} \mathrm{H}^{+}$ collisional coefficients were available. As they are expected to be higher than the present $\mathrm{He}-\mathrm{N}_{2} \mathrm{H}^{+}$ones, they would probably allow us to fit the observations with both a lower temperature and a lower density. In any case, the gas appears cooler than the dust $(\sim 12 \mathrm{~K})$ as estimated by Stepnik et al. (2003). Their resolution may be too coarse and a large fraction of the (warmer) envelope emission intermingled with the core emission, or their single temperature fit too simple by lack of constraints (we have shown that in the case of the L183 cold core, the $200 \mu \mathrm{m}$ emission does not trace the same dust as the submm emission and that a temperature gradient is compulsory, Pagani et al. 2004). Alternatively the gas and the dust being decoupled at these low densities $\left(n\left(\mathrm{H}_{2}\right) \leq 5 \times 10^{4} \mathrm{~cm}^{-3}\right.$ or less $)$, the dust could possibly be warmer than the gas though this is not usually expected.

The modeling of the $\mathrm{N}_{2} \mathrm{D}^{+}(J: 1-0)$ line implies a column density of 4 to $8 \times 10^{11} \mathrm{~cm}^{-2}$ depending on the chosen $\mathrm{N}_{2} \mathrm{D}^{+}$abundance profile, which we are unable to constrain with only one observation. The peak $\mathrm{N}_{2} \mathrm{H}^{+}$column density is $\sim 2 \times 10^{13} \mathrm{~cm}^{-2}$ and therefore the deuterium enrichment is 2-4\%. Compared to L183 (Pagani et al. 2007, 2009b), we identify a similar deuteration level at similar densities indicating that the evolutionary speed of the deuterium enrichment is probably similar and therefore can be used to determine the age of the core.

Beyond the core characterized by $\mathrm{C}^{18} \mathrm{O}$ narrow lines, the envelope, as traced by ${ }^{13} \mathrm{CO}$, is most accurately described by an almost constant density (in the range $200-1000 \mathrm{~cm}^{-3}$ ) and a large amount of turbulence $\left(V_{\text {turb }}(F W H M)=0.4 \mathrm{~km} \mathrm{~s}^{-1}\right)$. To follow its macroscopic velocity drift, we had to introduce a counterrotating pattern in the external layers of a constant velocity of $-0.3 \mathrm{~km} \mathrm{~s}^{-1}$. We do not claim that the ${ }^{13} \mathrm{CO}$ is indeed rotating but this is the only way to approach the velocity profile of this line with our model. Similarly, to reproduce the redshifted peak, we had to introduce an expansion velocity of $90 \mathrm{~m} \mathrm{~s}^{-1}$, which is comparable in amplitude to the infall velocity of the core. The fit is not perfect inasmuch as we were unable to centre the peak emission in our model on the same position as in the observed position-velocity diagram (Fig. 7) but the fit bears some ressemblance to reality as shown in Fig. 11. The derived parameters are therefore reasonably close to the true situation and this envelope is clearly decoupled from the core in terms of density profile as much as in terms of turbulence and large-scale motions. This region also has a very high abundance of ${ }^{13} \mathrm{CO}$ $\left(6 \times 10^{-6}\right)$ and an absence of $\mathrm{C}^{18} \mathrm{O}\left(<5 \times 10^{-9}\right.$, an abundance of $1 \times 10^{-8}$ is marginally consistant with the observations). The density is not well constrained in the external envelope but since the ${ }^{13} \mathrm{CO}$ abundance is already high (its standard value is $1-2 \times 10^{-6}$, i.e., 3 to 6 times less), the density cannot be lower than we have assumed here since a lower density would produce an even greater increase in the ${ }^{13} \mathrm{CO}$ abundance. In contrast, we could increase the density to lower the abundance of ${ }^{13} \mathrm{CO}$, but it would become higher than the density in the outermost $\mathrm{C}^{18} \mathrm{O}$ layers. In the absence of any additional ${ }^{13} \mathrm{CO}$ transitions, it is difficult to correctly constrain the density and abundance in these last, turbulent layers.
The collapsing core has a size of $\sim 3 \times 10^{4} \mathrm{AU}$ in radius and contains a mass of $\sim 4 M_{\odot}$. This size is larger than that of L1498, which was shown to be the largest by $50 \%$ of the nearby prestellar cores mentioned in the study by Shirley et al. (2005). Our mass estimate is somewhat approximate as the density of the $\mathrm{N}_{2} \mathrm{H}^{+}$region is possibly overestimated but on the other hand, the core could be slightly more extended than a sphere, and closer to a cylinder. The estimate derived from the dust observations is similar but more poorly constrained because of our lack of knowledge about the dust emissivity and temperature in the core on scales smaller than the PRONAOS resolution. The ${ }^{13} \mathrm{CO}$ low density filament contains at least $20 M_{\odot}$ accounting only for the part along its main axis that covers the core. Its diameter is $250000 \mathrm{AU}$ or $1.25 \mathrm{pc}$.

\section{Discussion}

Several properties of this area of the L1506 filament are remarkable and presently difficult to explain. The expanding and counter-drifting external envelope is one of them. One partially similar case has however been reported: Young et al. (1981) showed that the B5 core as traced by $\mathrm{C}^{18} \mathrm{O}$ had a position/velocity profile gradient that had an opposite sense to the ${ }^{12} \mathrm{CO}$ one, which they interpreted as a counter-rotating core (but they did not discuss the radial component). Contemporary theoretical work (Gillis et al. 1979; Mouschovias \& Paleologou 1979) was invoked to explain this situation: a radial, frozenin magnetic field perpendicular to the rotation axis could slow down the rotation of the cloud, but because the field remains anchored to the external regions, the magnetic torques do not vanish immediately and counter-rotation is induced in the core, which would be able to oscillate several times before coming to a halt. Whether this is truly the case here is unclear because 1) we are uncertain that the ${ }^{13} \mathrm{CO}$ itself traces rotation; 2) the expansion of the ${ }^{13} \mathrm{CO}$ envelope is not predicted by the model; and 3) we know nothing about the magnetic field inside this core (external parts have however been mapped, Moneti et al. 1984; Heiles 2000).

The strong and abrupt depletion of $\mathrm{C}^{18} \mathrm{O}$ at relatively low density is another remarkable property. This is similar to the L1498 case but in stark contrast to other very young cores, such as L1495B, L1521B, and L1521E, which exhibit no depletion despite a density that is typically 5 times higher (and possibly 10 times if $\mathrm{He}-\mathrm{N}_{2} \mathrm{H}^{+}$collisional coefficients indeed over-estimate the true density in L1506C or if we compare to the L1498 peak density derived by Shirley et al. 2005). Based mainly on this absence of depletion, these cores have been judged to be young but it is unusual that after recently acquiring their present density state, they show no kinematical sign of contraction. It is thus conceivable that they are older than assumed and that the absence of depletion could be due to another mechanism, such as strong desorption, an ill-understood mechanism (Roberts et al. 2007). It is understood that energetic cosmic ray impacts can desorb the most volatile ices such as $\mathrm{CO}$ (Leger et al. 1985; Hasegawa \& Herbst 1993; Bringa \& Johnson 2004; Roberts et al. 2007). The desorption efficiency is inversely correlated with the size of the grains because smaller grains are easier to warm up. Therefore, the question arises of whether or not cores such as L1495B have smaller grains than L1506C or not and what the difference in size of these grains should be to allow or prevent an efficient desorption of CO. As explained by Roberts et al. (2007), all desorption mechanisms remain poorly known and it is presently difficult to go further. However, if this explanation proves to be valid, then one has yet to 
understand why grains coagulate in some sources and not in others. Turbulence could be one of the main key parameters involved in this differentiation between cores. Based on the analyses of Chokshi et al. (1993) and Poppe \& Blum (1997) works, Flower et al. (2005) demonstrate that dust coagulation efficiency reaches a maximum for turbulent velocities in the range $10-80 \mathrm{~m} \mathrm{~s}^{-1}$ (see their Fig. A.1), a condition that is hardly achieved even in the most quiescent prestellar cores but fits the present case. In contrast, L1521E shows no depletion, while the turbulence is suprathermal (even for $\mathrm{N}_{2} \mathrm{H}^{+}, V_{\text {turb }}(F W H M)=$ $0.27 \mathrm{~km} \mathrm{~s}^{-1}$, Tafalla \& Santiago 2004, a factor of 4 at least larger than in L1506C). If the size of the grains in L1521E is not known, large grains have been advocated to explain submillimetre emission in L1506C. Stepnik et al. (2003) compiled a model where the very small grains, emitting at $60 \mu \mathrm{m}$, disappear for offsets lower than $r_{0}=4^{\prime}$ from the centre, while the submm emissivity increases by a factor of 3.4 because of the formation of fluffy aggregates. Their observations were taken with an angular resolution of $2-3.5^{\prime}$, so the value of $r_{0}$ could be lower and fit the extent $\left( \pm 200^{\prime \prime}\right)$ of the low turbulence region in L1506C. Grain growth, depletion, and desorption are certainly not completely understood at the moment.

The absence of $\mathrm{C}^{18} \mathrm{O}$ across 5/6th of the external envelope width as traced by the POM- $1{ }^{13} \mathrm{CO}$ observations is another puzzle. The ${ }^{13} \mathrm{CO}$ abundance itself seems to be higher than usual so that the ${ }^{13} \mathrm{CO} / \mathrm{C}^{18} \mathrm{O}$ ratio reaches $600-1200$ over a large region. Although ${ }^{13} \mathrm{CO}$ fractionation does exist, it is weak and does not seem to be able to explain this difference. More observations of the envelope (including ${ }^{13} \mathrm{CO}(J: 1-0)$ and $(J: 2-1)$ and very low noise $\mathrm{C}^{18} \mathrm{O}$ transitions) are needed to address this issue.

Clear traces of collapse among prestellar cores was found in a few cases. Lee et al. (1999, 2004) observed a large number of asymmetrically blue profiles towards a sample of starless cores but they traced the CS kinematics with respect to $\mathrm{N}_{2} \mathrm{H}^{+}$or $\mathrm{DCO}^{+}$and, since CS is depleted inside the $\mathrm{N}_{2} \mathrm{H}^{+}$region, they traced the inward motion of the envelope only. A few prestellar cores are indeed modeled with a collapsing core, the most well studied being L1544 (e.g., Williams et al. 1999; Tafalla et al. 2002; van der Tak et al. 2005), which was reported to clearly undergo a collapse from line profile fitting. Tafalla et al. (2004) also invoke the possibility of infall from the observations of two other cores, L1498 and L1517B despite the absence of a clear velocity gradient and Williams et al. (2006) found that L694-2 has a profile reminiscent of L1544, which is also indicative of probable infall. This was confirmed by Lee et al. (2007) who also reported the infall of L1197. Here the amplitude of the inward motion of $\mathrm{N}_{2} \mathrm{H}^{+}$is not strongly constrained but is clearly necessary to reproduce the velocity-profile plot: no infall at all is clearly the wrong result but the details of the radial velocity profile are uncertain. The inward motion is also clearly indicated by the $\mathrm{C}^{18} \mathrm{O}$ and $\mathrm{C}^{17} \mathrm{O}$ lines. It is the first time to our knowledge that these lines trace the collapse of a core. We understand that this is possible here because several favorable factors are met:

1. No CO isotopologues are present in the central part of the core, which clearly separates the front and rear moving layers as indicated by $\mathrm{C}^{17} \mathrm{O}$.

2. No $\mathrm{C}^{18} \mathrm{O}$ in the extended envelope, which would have either hidden or blurred the faint variation in intensity between the blue and red emission that enable us to differentiate between expansion and contraction.
3. Low $\mathrm{C}^{18} \mathrm{O}$ velocity dispersion, which increases the line opacity near unity $\left(\tau_{J: 1-0}=0.56, \tau_{J: 2-1}=0.91\right)$. Combined with the outwardly decreasing excitation temperature, it allows for weak but measurable differential absorption between the blue and red components.

The most surprising aspect is that this core with a density of lower than $\sim 1 \times 10^{5} \mathrm{~cm}^{-3}$ is supposed to be dynamically stable given its total mass (less than $10 M_{\odot}$, Keto \& Caselli 2008), which is clearly not the case here (the core mass being estimated to be $\sim 4 M_{\odot}$ ). However, as noted by Keto \& Caselli (2008), most clouds without non-thermal energy support would be unstable and, in the present case, turbulence is abnormally low, non-measurable in several parts of the core. This would support the idea of a possible collapse though we have no information about the magnetic field intensity and direction. If a magnetic field is indeed present and induces the core counter-rotation, it is not incompatible with infall because it is included in the magnetic braking model of Mouschovias \& Paleologou (1979). Oscillations or sound waves were invoked in the case of e.g., B68 (Maret et al. 2007); one could be tempted to invoke a similar argument here but the rotation of $\mathrm{C}^{18} \mathrm{O}$ and "counter-rotation" of ${ }^{13} \mathrm{CO}$ seem difficult to explain in terms of sound waves.

\section{Conclusions}

The large size of L1506C and its low density clearly indicate that it is not yet a prestellar core but its inward motion and its kinematical decoupling from the outer envelope are signs that it is in the process of turning into one. It is the first time that a low density core is clearly observed to collapse to form a prestellar core, both the kinematical and density status of L1498 being somewhat unclear. Two different evolutionary paths from normal clouds to prestellar cores seem to exist: one that begins with density enhancement first much before any depletion occurs and one that begins with depletion first. Two questions arise: what is the reason for this differentiation (turbulence via its action on grain growth, itself limiting the desorption capability of cosmic rays) and do both paths really produce standard prestellar cores? Coming observations with the Herschel Space Observatory will help us to address these questions and the nature of the dust inside this object.

Acknowledgements. We want to thank an anonymous referee for her/his fruitful comments which helped to improve the manuscript and P.F. Goldsmith for fruitful discussions. This research has made use of the SIMBAD database, operated at CDS, Strasbourg, France.

\section{References}

Abergel, A., Boulanger, F., Mizuno, A., \& Fukui, Y. 1994, ApJ, 423, L59 Bergin, E. A., \& Langer, W. D. 1997, ApJ, 486, 316

Bergin, E. A., Maret, S., van der Tak, F. F. S., et al. 2006, ApJ, 645, 369 Bernard, J. P., Boulanger, F., Desert, F. X., \& Puget, J. L. 1992, A\&A, 263, 258 Bernes, C. 1979, A\&A, 73, 67

Bohlin, R. C., Savage, B. D., \& Drake, J. F. 1978, ApJ, 224, 132

Bringa, E. M., \& Johnson, R. E. 2004, ApJ, 603, 159

Caselli, P. 2002, Planet. Space Sci., 50, 1133

Chokshi, A., Tielens, A. G. G. M., \& Hollenbach, D. 1993, ApJ, 407, 806

Daniel, F., Dubernet, M.-L., Meuwly, M., Cernicharo, J., \& Pagani, L. 2005, MNRAS, 363, 1083

Dupac, X., \& Giard, M. 2002, MNRAS, 330, 497

Elias, J. H. 1978, ApJ, 224, 857

Flower, D. R., Pineau Des Forêts, G., \& Walmsley, C. M. 2005, A\&A, 436, 933 Gillis, J., Mestel, L., \& Paris, R. B. 1979, MNRAS, 187, 311 
A\&A 512, A3 (2010)

Hasegawa, T. I., \& Herbst, E. 1993, MNRAS, 261, 83

Heiles, C. 2000, AJ, 119, 923

Hirota, T., Ito, T., \& Yamamoto, S. 2002, ApJ, 565, 359

Hirota, T., Maezawa, H., \& Yamamoto, S. 2004, ApJ, 617, 399

Kenyon, S. J., Dobrzycka, D., \& Hartmann, L. 1994, AJ, 108, 1872

Keto, E., \& Caselli, P. 2008, ApJ, 683, 238

Klapper, G., Surin, L., Lewen, F., et al. 2003, ApJ, 582, 262

Lada, C. J., Huard, T. L., Crews, L. J., \& Alves, J. F. 2004, ApJ, 610, 303

Lee, C. W., \& Myers, P. C. 1999, ApJS, 123, 233

Lee, C. W., Myers, P. C., \& Tafalla, M. 1999, ApJ, 526, 788

Lee, C. W., Myers, P. C., \& Plume, R. 2004, ApJS, 153, 523

Lee, S. H., Park, Y.-S., Sohn, J., Lee, C. W., \& Lee, H. M. 2007, ApJ, 660, 1326

Leger, A., Jura, M., \& Omont, A. 1985, A\&A, 144, 147

Maret, S., Bergin, E. A., \& Lada, C. J. 2007, ApJ, 670, L25

Moneti, A., Pipher, J. L., Helfer, H. L., McMillan, R. S., \& Perry, M. L. 1984, ApJ, 282, 508

Mouschovias, T. C., \& Paleologou, E. V. 1979, ApJ, 230, 204

Myers, P. C., Mardones, D., Tafalla, M., Williams, J. P., \& Wilner, D. J. 1996, ApJ, 465, L133+

Nercessian, E., Castets, A., Benayoun, J. J., \& Cernicharo, J. 1988, A\&A, 189, 207

Onishi, T., Mizuno, A., Kawamura, A., Ogawa, H., \& Fukui, Y. 1996, ApJ, 465, 815

Ossenkopf, V., \& Henning, T. 1994, A\&A, 291, 943
Pagani, L., \& Breart de Boisanger, C. 1996, A\&A, 312, 989 Pagani, L., Bacmann, A., Motte, F., et al. 2004, A\&A, 417, 605 Pagani, L., Pardo, J.-R., Apponi, A. J., Bacmann, A., \& Cabrit, S. 2005, A\&A, 429, 181

Pagani, L., Bacmann, A., Cabrit, S., \& Vastel, C. 2007, A\&A, 467, 179

Pagani, L., Daniel, F., \& Dubernet, M.-L. 2009a, A\&A, 494, 719

Pagani, L., Vastel, C., Hugo, E., et al. 2009b, A\&A, 494, 623

Poppe, T., \& Blum, J. 1997, Adv. Space Res., 20, 1595

Roberts, J. F., Rawlings, J. M. C., Viti, S., \& Williams, D. A. 2007, MNRAS, 382,733

Shirley, Y. L., Nordhaus, M. K., Grcevich, J. M., et al. 2005, ApJ, 632, 982

Stepnik, B., Abergel, A., Bernard, J.-P., et al. 2003, A\&A, 398, 551

Tafalla, M., \& Santiago, J. 2004, A\&A, 414, L53

Tafalla, M., Myers, P. C., Caselli, P., Walmsley, C. M., \& Comito, C. 2002, ApJ, 569,815

Tafalla, M., Myers, P. C., Caselli, P., \& Walmsley, C. M. 2004, A\&A, 416, 191 van der Tak, F. F. S., Caselli, P., \& Ceccarelli, C. 2005, A\&A, 439, 195

Willacy, K., Langer, W. D., \& Velusamy, T. 1998, ApJ, 507, L171

Williams, J. P., Myers, P. C., Wilner, D. J., \& di Francesco, J. 1999, ApJ, 513, L61

Williams, J. P., Lee, C. W., \& Myers, P. C. 2006, ApJ, 636, 952

Young, J. S., Goldsmith, P. F., Langer, W. D., \& Wilson, R. W. 1981, ApJ, 251, L81

Zucconi, A., Walmsley, C. M., \& Galli, D. 2001, A\&A, 376, 650 